\title{
EXCURSION SETS OF THREE CLASSES OF STABLE RANDOM FIELDS
}

\author{
ROBERT J. ADLER, ${ }^{*}$ Technion \\ GENNADY SAMORODNITSKY,** Cornell University \\ JONATHAN E. TAYLOR, ${ }^{* * *}$ Stanford University
}

\begin{abstract}
Studying the geometry generated by Gaussian and Gaussian-related random fields via their excursion sets is now a well-developed and well-understood subject. The purely non-Gaussian scenario has, however, not been studied at all. In this paper we look at three classes of stable random fields, and obtain asymptotic formulae for the mean values of various geometric characteristics of their excursion sets over high levels. While the formulae are asymptotic, they contain enough information to show that not only do stable random fields exhibit geometric behaviour very different from that of Gaussian fields, but they also differ significantly among themselves.
\end{abstract}

Keywords: Stable random field; harmonisable field; excursion set; Euler characteristic; intrinsic volume; geometry

2010 Mathematics Subject Classification: Primary 60G52

Secondary 60D05; 60G10; 60G60

\section{Introduction}

We are interested in the structure of the sample paths of certain smooth stable random fields $f: \mathbb{R}^{N} \rightarrow \mathbb{R}, N \geq 1$. We shall study these through certain geometric properties of their excursion sets

$$
A_{u} \equiv A_{u}(f, M):=\{t \in M: f(t) \geq u\},
$$

where $M \subset \mathbb{R}^{N}$ and $u \in \mathbb{R}$.

Excursion sets have been widely studied for Gaussian and Gaussian-related random fields. Their applications appear in disciplines as widespread as astrophysics and medical imaging, where they have also been used in a variety of hypothesis testing situations. A good introductory reference to the applications is still Keith Worsley's exposition [9], although [4], when ready,

Received 24 February 2009; revision received 19 January 2010.

* Postal address: Faculty of Electrical Engineering, Technion, Haifa, 32000, Israel.

Email address: robert@ee.technion.ac.il

Research supported in part by the US-Israel Binational Science Foundation, grant number 2008262, and the NSF, grant number DMS-0852227.

** Postal address: Operations Research and Information Engineering, Cornell University, Ithaca, NY 14853, USA.

Email address: gs18@ cornell.edu

Research supported in part by the US-Israel Binational Science Foundation, grant number 2008262, by an NSA grant MSPF-05G-049, and an ARO grant W911NF-07-1-0078 at Cornell University.

*** Postal address: Department of Statistics, Stanford University, Stanford, CA 94305-4065, USA.

Email address: jonathan.taylor@stanford.edu

Research supported in part by the US-Israel Binational Science Foundation, grant number 2008262, by NSF grants DMS-0405970, 0852227, 0906801, and the Natural Sciences and Engineering Research Council of Canada. 
will have a lot more detail. On the more theoretical side, where excursion sets are seen to generate an elegant geometric structure, our basic reference will be the recent monograph [2].

The Gaussian and Gaussian-related scenarios allow for the development of explicit formulae for the expectations of many of the geometrical quantifiers of excursion sets. Unfortunately, we cannot expect that the same will occur in the stable case, for which our basic reference will be [8]. Here, explicit formulae for even the marginal densities of $f$ are unknown, although much is known about their asymptotics. Indeed, when we began this research, we expected to be able to find little beyond some asymptotic formulae relating to the excursion sets generated by stable fields that might mimic the Gaussian ones, much as we did for level crossings of stable processes on the real line, in [1] and [3].

What we found turned out to be far more interesting. It is well known that the structure of stable processes is far more complicated than that of their Gaussian counterparts. For example, whereas in the Gaussian case many stationary processes have both a moving average representation (with respect to white noise) as well as a harmonisable representation, in the stable case moving average and harmonisable processes belong to quite distinct families. These differences are well understood in terms of mathematical structure, but it turns out that the sample path distinctions between different classes of stable processes become significantly highlighted by looking at the excursion sets that they generate, primarily in the multidimensional setting.

This paper has two aims. One is to provide explicit, albeit asymptotic, formulae for the expectations of the Euler characteristics (defined below) and other geometric quantifiers of excursion sets generated by stable random fields. These, we believe, will find immediate application in random field modelling using these processes. The second is to better understand the differences between various stable random fields via their excursion sets.

The remainder of the paper is structured as follows. In the next section we shall define the Euler characteristic and related Lipschitz-Killing curvatures. In Section 3 we give a description of the main result from the Gaussian theory, which is a precise formula for the expected value of the Lipschitz-Killing curvatures of Gaussian excursion sets. With this in hand, in Sections 4-6 we start with the new results, for sub-Gaussian, harmonisable, and concatenated-harmonisable random fields, deriving asymptotic formulae for the expected values of the Euler characteristics of their excursion sets. In Section 7 we show how to lift these results to all the Lipschitz-Killing curvatures, at least for isotropic fields. A technical appendix completes the paper.

\section{Euler characteristics and Lipschitz-Killing curvatures}

Throughout this paper, our parameter sets $M$ will be taken to be convex subsets of $\mathbb{R}^{N}$, and, for much of it, we shall restrict ourselves to $N$-dimensional rectangles. In these cases we shall always write $T$ rather than $M$, where $T$ is given by

$$
T:=\prod_{j=1}^{N}\left[0, T_{j}\right] .
$$

For convex sets in $\mathbb{R}^{N}$, and for excursion sets of smooth functions defined over them, there are $N+1$ functionals which describe their geometry. These are known under a variety of names, including Minkowski functionals, intrinsic volumes, quermassintegrals, and LipschitzKilling curvatures, being related to one another by differences in the way they are ordered or normalized. For consistency with [2], which we shall use heavily when we wish to cite a result 
without proof, we shall work with Lipschitz-Killing curvatures. Perhaps the easiest way to define these is via Steiner's formula, a classic result of integral geometry.

To state Steiner's formula, let $M$ be a convex set of dimension $N$, sitting in $\mathbb{R}^{N^{\prime}}$, where $N^{\prime} \geq N$. (For example, $M$ is a two-dimensional disk sitting in $\mathbb{R}^{3}$.) The tube of radius $\rho$ around $M$ is defined to be

$$
\operatorname{Tube}(M, \rho)=\left\{x \in \mathbb{R}^{N^{\prime}}: \inf _{y \in M}|x-y| \leq \rho\right\} .
$$

With $\lambda_{N}$ denoting the Lebesgue measure in $\mathbb{R}^{N}$, Steiner's formula states that there is an exact polynomial expansion of order $N$ for the $\lambda_{N^{\prime}}$ measure of $\operatorname{Tube}(M, \rho)$ given by

$$
\lambda_{N^{\prime}}(\operatorname{Tube}(M, \rho))=\sum_{j=0}^{N} \omega_{N^{\prime}-j} \rho^{N^{\prime}-j} \mathcal{L}_{j}(M),
$$

where

$$
\omega_{j}=\frac{\pi^{j / 2}}{\Gamma(j / 2+1)}
$$

is the volume of the unit ball in $\mathbb{R}^{j}$. The numbers $\mathcal{L}_{j}(M)$ are the Lipschitz-Killing curvatures of $M$. Steiner's formula has been significantly extended since its original formulation, and a version of it holds, for example, when $M$ is a locally convex, sufficiently smooth, stratified manifold. In this case, in which the result is known as Weyl's formula, (2) holds for only sufficiently small $\rho$, but this suffices to uniquely define the Lipschitz-Killing curvatures. We shall return to this point later.

The $\mathscr{L}_{j}$ scale nicely, in the sense that, for $\lambda>0, \mathcal{L}_{j}(\lambda M)=\lambda^{j} \mathcal{L}_{j}(M)$. As is obvious from (2), $\mathscr{L}_{N}(M)$ measures the volume of $M, \mathscr{L}_{N-1}(M)$ is related to its surface measure, etc. The last one, $\mathscr{L}_{0}(M)$, is the Euler characteristic of $M$ which, since we shall use it very often, we also denote by $\varphi(M)$. Of all of the $\mathcal{L}_{j}(M)$, the Euler characteristic is the only one that does not change under smooth deformations of $M$. If $M$ is one dimensional then $\varphi(M)$ counts the number of connected components in $M$. If $M$ is two dimensional then it counts the number of connected components minus the number of holes. In three dimensions, it counts the number of connected components minus the number of 'handles' plus the number of holes.

We note, for later usage, that the Lipschitz-Killing curvatures of the $N$-rectangle (1) are given by

$$
\mathcal{L}_{j}(T)=\mathcal{L}_{j}\left(\prod_{i=1}^{N}\left[0, T_{i}\right]\right)=\sum T_{i_{1}} \cdots T_{i_{j}},
$$

where the sum is taken over the $\left(\begin{array}{c}N \\ j\end{array}\right)$ distinct choices of subscripts $i_{1}, \ldots, i_{j}$.

There is another way to write (3), which will be useful later on. Let $\mathcal{O}_{j} \equiv \mathcal{O}_{j}(T)$ denote the collection of the $\left(\begin{array}{c}N \\ j\end{array}\right) j$-dimensional facets of $T$ which contain the origin. (Thus, for example, $\mathcal{O}_{N}$ is $T$ itself, while $\mathcal{O}_{1}$ contains the $N$ one-dimensional edges of $T$ lying on the positive axes $e_{1}, \ldots, e_{N}$ of $\mathbb{R}^{N}$.) Furthermore, if $J$ is a facet in $\mathcal{O}_{j}$, let $|J|$ denote its $j$-dimensional Lebesgue measure. Then it is immediate from (3) that

$$
\mathcal{L}_{j}(T)=\sum_{J \in \mathcal{O}_{j}}|J| .
$$


The Lipschitz-Killing curvatures play a central role in much of integral geometry, but for the moment we shall note only one of their properties, known as Hadwiger's theorem [6]. Suppose that we have a functional $\psi$ on compact convex sets that is additive, in the sense that, if $A, B$, and $A \cup B$ are compact convex, then

$$
\psi(A \cup B)=\psi(A)+\psi(B)-\psi(A \cap B) .
$$

If it is also true that $\psi$ is invariant under rigid motions and continuous in the Hausdorff metric, then there are ( $\psi$-dependent) constants such that

$$
\psi(A)=\sum_{j=0}^{N} c_{j} \mathcal{L}_{j}(A)
$$

Thus, studying intrinsic volumes is equivalent to studying a far wider class of functionals on sets. Our aim is to study the Lipschitz-Killing curvatures of excursion sets.

\section{Gaussian excursion set geometry}

In this section we want to summarise some results about the excursion sets of Gaussian random fields. There are two reasons for doing this. The first is that it gives us a basis to which to compare the results of this paper for stable random fields, and the second is that, in all the cases that we shall consider in this paper, the proof for the stable case follows from the Gaussian case and a conditioning argument.

To state the main result for the Gaussian case, we need a little notation, for which we now assume that $f$ is a mean 0 , stationary, Gaussian random field on $\mathbb{R}^{N}$ with constant variance $\sigma^{2}$. Assuming that $f$ is also almost surely (a.s.) $C^{2}$, we define the second-order spectral moments

$$
\lambda_{i j}=\mathrm{E}\left\{\frac{\partial f(t)}{\partial t_{i}} \frac{\partial f(t)}{\partial t_{j}}\right\} .
$$

With $\left\{e_{1}, \ldots, e_{N}\right\}$ denoting the positive axes of $\mathbb{R}^{N}$, suppose that $J \in \mathcal{O}_{j}$ is a $j$-dimensional facet of a rectangle $T$. We write $\Lambda_{J}$ for the matrix

$$
\Lambda_{J}:=\left\{\lambda_{i j}: i, j \in \sigma(J)\right\}
$$

where

$$
\sigma(J):=\left\{j: e_{j} \cap(J \backslash\{0\}) \neq \varnothing\right\} .
$$

Note that if $f$ is isotropic then there is a constant, which we shall write as $\lambda_{2}$, such that

$$
\lambda_{i j}= \begin{cases}\lambda_{2}, & i=j, \\ 0, & i \neq j .\end{cases}
$$

Next, we need the Hermite polynomials

$$
H_{n}(x)=n ! \sum_{j=0}^{\lfloor n / 2\rfloor} \frac{(-1)^{j} x^{n-2 j}}{j !(n-2 j) ! 2^{j}}, \quad n \geq 0, x \in \mathbb{R},
$$

where $\lfloor a\rfloor$ is the largest integer less than or equal to $a$, and, for notational convenience, we 
define

$$
H_{-1}(x)=\sqrt{2 \pi} \Psi(x) \mathrm{e}^{x^{2} / 2},
$$

where

$$
\Psi(x):=\frac{1}{\sqrt{2 \pi}} \int_{x}^{\infty} \mathrm{e}^{-u^{2} / 2} \mathrm{~d} u .
$$

We also adopt the notation

$$
\rho_{n}(u)=(2 \pi)^{-(n+1) / 2} H_{n-1}(u) \mathrm{e}^{-u^{2} / 2}, \quad n \geq 0 .
$$

The following is a combination of Theorem 11.7.2 and the discussion in Section 11.8 of [2].

Theorem 1. Let $f$ be a zero-mean, stationary Gaussian field on an $N$-rectangle $T$ with variance $\sigma^{2}$ and a.s. $C^{2}$ sample paths, and such that the joint distribution of $f$ and its first and second derivatives at each point $t \in T$ is nondegenerate. Suppose that the joint modulus of continuity $\omega(\eta)$ of all the second-order partial derivatives of $f$ satisfies

$$
\mathrm{P}\{\omega(\eta)>\varepsilon\}=o\left(\eta^{N}\right) \text { as } \eta \downarrow 0
$$

for all $\varepsilon>0$. Then the mean value of the Euler characteristic of its excursion set is given by

$$
\mathrm{E}\left\{\varphi\left(A_{u}(f, T)\right)\right\}=\sum_{n=0}^{N} \sum_{J \in \mathcal{O}_{n}} \frac{|J|\left|\operatorname{det}\left(\Lambda_{J}\right)\right|^{1 / 2}}{\sigma^{n}} \rho_{n}\left(\frac{u}{\sigma}\right) .
$$

Furthermore, if $f$ is isotropic and $M$ compact and convex, then, for all $0 \leq j \leq N$,

$$
\mathrm{E}\left\{\mathcal{L}_{j}\left(A_{u}(f, M)\right)\right\}=\sum_{n=0}^{N-j}\left[\begin{array}{l}
N \\
n
\end{array}\right] \mathcal{L}_{n+j}(M) \rho_{n}\left(\frac{u}{\sigma}\right)\left(\frac{\lambda_{2}}{\sigma^{2}}\right)^{(n+j) / 2},
$$

where $\lambda_{2}$ is as in (8) and

$$
\left[\begin{array}{c}
N \\
j
\end{array}\right]=\left(\begin{array}{c}
N \\
j
\end{array}\right) \frac{\omega_{N}}{\omega_{N-j} \omega_{j}} .
$$

In fact, results of this nature hold in much wider generality, when $M$ is a general stratified manifold $M$ and $f$ is neither stationary nor isotropic. We refer the interested reader to Chapters 12 and 13 of [2].

One observation that follows from (10) and (11) comes by rewriting the sums as power series in $u$, from which we immediately see that the leading-order terms, of orders $u^{N-1}$ and $u^{N-j-1}$, respectively, are associated with the volume of $T$ or $M$, while lower-order terms are associated with the other Lipschitz-Killing curvatures. This observation will be important when it comes to understanding the stable case.

Finally, although it will not be important in what follows, we note that condition (9) is an extremely mild condition for $C^{2}$ Gaussian processes, and is easily checked from the covariance function of the process. 


\section{Sub-Gaussian fields}

With the Gaussian case behind us, we shall now look at what is probably the simplest of all stable random fields, the sub-Gaussian fields. Despite their simplicity, we shall see that their behaviour is already very different from the Gaussian case.

To define these processes, we let $g$ be a Gaussian random field on $M$, and, for some $\alpha \in(0,2)$, let $X$ be an $S_{\alpha / 2}\left(\sigma_{\alpha}, 1,0\right)$ random variable independent of $g$ (see [8, p. 142] for notation), where

$$
\sigma_{\alpha}:=\cos \left(\frac{\pi \alpha}{4}\right)^{2 / \alpha}
$$

Thus, $X$ is a positive, strictly $\alpha / 2$-stable random variable with Laplace transform

$$
\mathrm{E}\left\{\mathrm{e}^{-t X}\right\}=\mathrm{e}^{-t^{\alpha / 2}}, \quad t>0 .
$$

Taking $X$ independent of $g$ and setting

$$
f(t)=X^{1 / 2} g(t)
$$

defines a sub-Gaussian random field.

To state our first result, we need some notation. For any functions $a, b: \mathbb{R} \rightarrow \mathbb{R}$, we write

$$
a \asymp b \quad \Longleftrightarrow \quad \lim _{u \rightarrow \infty} \frac{a(u)}{b(u)}=1
$$

Theorem 2. Let $g$ be a zero-mean, stationary Gaussian field on the $N$-rectangle $T$, satisfying the conditions of Theorem 1. Denote its variance by $\sigma_{g}^{2}$ and the matrices of its second-order spectral moments by $\Lambda_{J}$, as in (7). Let $f$, as above, be the sub-Gaussian field $X^{1 / 2} g$. Then

$$
\mathrm{E}\left\{\varphi\left(A_{u}(f, T)\right)\right\} \asymp u^{-\alpha}\left(K_{0}+\sum_{n=1}^{N} K_{n} \sum_{J \in \mathcal{O}_{n}}|J|\left|\Lambda_{J}\right|^{1 / 2}\right),
$$

where we write $\left|\Lambda_{J}\right|$ for $\left|\operatorname{det}\left(\Lambda_{J}\right)\right|$,

$$
K_{0}=\frac{2^{-1+\alpha / 2} \sigma_{g}^{\alpha} \Gamma((\alpha+1) / 2)}{\sqrt{\pi} \Gamma(1-\alpha / 2)},
$$

and, for $n \geq 1$,

$$
K_{n}=\frac{\alpha 2^{-1+\alpha / 2} \sigma_{g}^{\alpha} \Gamma((\alpha+1) / 2)}{\Gamma(1-\alpha / 2)} \frac{(n-1) !}{\pi^{(n+1) / 2} \sigma_{g}^{n}} \sum_{j=0}^{\lfloor(n-1) / 2\rfloor} \frac{(-1)^{j} \Gamma((\alpha+n-1-2 j) / 2)}{2^{2 j+1} j !(n-1-2 j) !} .
$$

If $g$ (and so $f$ ) is isotropic, with second spectral moment $\lambda_{2}(c f .(8))$ and $M$ is a compact convex domain, then

$$
\mathrm{E}\left\{\varphi\left(A_{u}(f, M)\right)\right\} \asymp u^{-\alpha} \sum_{n=0}^{N} K_{n} \lambda_{2}^{n / 2} \mathcal{L}_{n}(M)
$$


Under isotropy, a corresponding result holds for the expected Lipschitz-Killing curvatures of other orders as well. We shall look at this later, in Section 7.

Before proving this theorem, we shall take a moment to see how very different it is from the purely Gaussian case, despite the fact that the excursion sets of $f$ and $g$ are very simply related by the fact that

$$
A_{u}(f, T)=A_{u X^{-1 / 2}}(g, T) .
$$

Consider the case in which $N=1$, when the theorem relates to an $f$ defined over the interval $[0, T]$. Then

$$
\varphi\left(A_{u}(f,[0, T])\right)=\mathbf{1}_{\{f(0) \geq u\}}+C_{u}(f, T),
$$

where $C_{u}(f, T)$ is the number of upcrossings of the level $u$ by $f$ in $[0, T]$. Taking expectations, we see that the two terms that appear in (12) correspond to (asymptotics for) $\mathrm{E}\left\{\mathbf{1}_{\{f(0) \geq u\}}\right\}=$ $\mathrm{P}\{f(0) \geq u\}$ and

$$
\mathrm{E}\left\{C_{u}(f, T)\right\} \asymp u^{-\alpha} \frac{2^{-1+\alpha / 2} \Gamma(1+\alpha / 2)\left(\lambda_{11}\right)^{1 / 2} T}{\pi \Gamma(1-\alpha / 2) \sigma_{g}^{(1-\alpha) / 2}},
$$

where the asymptotics here come from either substitution into (12) or Theorem 3.2 of [3], in which one-dimensional level crossings are studied. Note that both terms, i.e. the 'boundary' and 'interior' terms, have the same asymptotics, of the form $u^{-\alpha}$. This is also true when looking at the case of general $N$ in (12), in that facets of $T$ of all dimensions contribute to the asymptotics, and this is probably the most interesting aspect of the result.

Recall that in the Gaussian case we saw that in an expansion of the mean Euler characteristic the leading term involved only the volume of $T$, with the surface area affecting only the second and later terms, etc. In the sub-Gaussian case, however, it is clear from (12) that the full geometry of $T$ affects the first term of any such expansion.

A heuristic explanation for this is easy to find. In the Gaussian case, if the level $u$ is high, the excursion set will, with high probability, contain only a small set which will be unlikely to intersect the boundary of $T$. Hence, only volume terms appear in the highest-order term when expanding $\mathrm{E}\left\{\varphi\left(A_{u}(f, T)\right)\right\}$. However, in the notation of the theorem, an excursion set of the sub-Gaussian $f$ at the level $u$ has the same geometry as an excursion set of the Gaussian $g$ at the level $u / \sqrt{X}$. Although $u$ may be large, the most likely reason for $f$ to reach this level is that $X$ is also large and that $u / \sqrt{X}$ is roughly $O(1)$. This being the case, $A_{u / \sqrt{X}}(g, T)$ is a 'typical' rather than 'rare' excursion set for $g$, and so has a reasonable probability of meeting the lower-dimensional facets of $T$. Thus, it is not surprising that they contribute to (12).

Despite the (hopefully) convincing tone of these heuristics, the proof follows a different, and purely analytic, route.

Proof of Theorem 2. We shall start with the general, nonisotropic case, and $T$ a rectangle.

As noted above, $A_{u}(f, T)=A_{u X^{-1 / 2}}(g, T)$. Thus, it is immediate that $\varphi\left(A_{u}(f, T)\right)$ is well defined since the same is true of $\varphi\left(A_{u}(g, T)\right)$ for every $u$. Conditioning on $X$, we should now like to claim that

$$
\mathrm{E}\left\{\varphi\left(A_{u}(f, T)\right)\right\}=\mathrm{E}\left\{\mathrm{E}\left\{\varphi\left(A_{u}(f, T)\right)\right\} \mid X\right\},
$$

and then to use the Gaussian theorem, Theorem 1, to compute the inner expectation. However, to justify this, we need to establish two facts. The first is that the conditioned process, $f \mid X$, 
which is clearly Gaussian, satisfies all the conditions of Theorem 1. This is trivial, since $g$ is assumed to satisfy these conditions and $f \mid X$ is no more than a constant multiple of $g$.

The trickier problem is that to apply the iterated expectation in (14), we need to know, a priori, that the absolute moment $\mathrm{E}\left\{\left|\varphi\left(A_{u}(f, T)\right)\right|\right\}$ is finite. Since this is technical, we shall leave it to Lemma 3 in Appendix A, and for the moment progress assuming that it is true.

Then, applying Theorem 1 to (14), we have

$$
\begin{aligned}
\mathrm{E}\left\{\varphi\left(A_{u}(f, T)\right)\right\} & =\mathrm{E}\left\{\mathrm{E}\left\{\varphi\left(A_{u / \sqrt{X}}(g, T)\right) \mid X\right\}\right\} \\
& =\sum_{n=0}^{N} \mathrm{E}\left\{H_{n-1}\left(\frac{u}{\sigma_{g} \sqrt{X}}\right) \mathrm{e}^{-u^{2} / 2 \sigma_{g}^{2} X}\right\} \sum_{J \in \mathcal{O}_{n}} \frac{|J|\left|\Lambda_{J}\right|^{1 / 2}}{(2 \pi)^{(n+1) / 2} \sigma_{g}^{n}} .
\end{aligned}
$$

To evaluate this triple sum (the third sum appears implicitly in the Hermite polynomials), we need to consider typical terms of the form

$$
\mathrm{E}\left\{u^{k}\left(\sigma_{g}^{2} X\right)^{-k / 2} \mathrm{e}^{-u^{2} / 2 \sigma_{g}^{2} X}\right\}=\left(\frac{u}{\sigma_{g}}\right)^{k} \mathrm{E}\left\{X^{-k / 2} \mathrm{e}^{-u^{2} / 2 \sigma_{g}^{2} X}\right\}
$$

and the one atypical term, coming from $n=0$ and $H_{-1}$, of the form

$$
\mathrm{E}\left\{\Psi\left(\frac{u}{\sigma_{g} \sqrt{X}}\right)\right\}
$$

The asymptotics of these expressions is covered in Lemma 1, below, which will be crucial to most of the computations of this section. Once we prove Lemma 1, (12) is the consequence of a little algebra, and so the first part of the theorem is established.

As for the isotropic case, note that taking $M \equiv T$, (13) follows immediately from (12) on noting (4) and the fact that $\operatorname{det}\left(\Lambda_{J}\right)=\lambda_{2}^{\operatorname{dim} J}$. To establish the argument for general convex compact $M$, we use this fact and Hadwiger's result (6).

To this end, define a functional $\psi$ on convex compact sets $M \subset \mathbb{R}^{N}$ by setting

$$
\psi(M):=\mathrm{E}\left\{\varphi\left(A_{u}(f, M)\right)\right\} .
$$

It is immediate that $\psi$ is additive (in the sense of (5)) and, using the same general arguments as in Appendix A, by bounding $\varphi\left(A_{u}(f, M)\right)$ by the number of critical points of $f$ over $M$, that $\psi$ is also continuous over convex compact sets. Furthermore, if $g$ is isotropic, it follows that $\psi$ is also invariant under rigid motions. Thus, Hadwiger's result applies and we find that there exist constants $c_{j}$ such that, for convex $M$,

$$
\mathrm{E}\left\{\varphi\left(A_{u}(f, M)\right)\right\}=\sum_{j=0}^{N} c_{j} \mathcal{L}_{j}(M) .
$$

Since the $c_{j}$ are not dependent on $M$, the fact that (13) holds for rectangles defines them, and this fact and the above equation imply that (13) holds for convex $M$ as well, as required.

Lemma 1. Let $X$ be as in Theorem 2. Then, for any $\beta>-\alpha$,

$$
\lim _{u \rightarrow \infty} u^{\alpha+\beta} \mathrm{E}\left\{X^{-\beta / 2} \mathrm{e}^{-u^{2} / 2 \sigma_{g}^{2} X}\right\}=2^{(\alpha+\beta-2) / 2} \alpha C_{\alpha / 2} \sigma_{\alpha}^{\alpha / 2} \sigma_{g}^{\alpha+\beta} \Gamma\left(\frac{\alpha+\beta}{2}\right) .
$$


Furthermore,

$$
\lim _{u \rightarrow \infty} u^{\alpha} \mathrm{E}\left\{\Psi\left(\frac{u}{\sigma_{g} \sqrt{X}}\right)\right\}=2^{-1+\alpha / 2} \pi^{-1 / 2} C_{\alpha / 2} \sigma_{\alpha}^{\alpha / 2} \sigma_{g}^{\alpha} \Gamma\left(\frac{1+\alpha}{2}\right) .
$$

Proof. The limit (15) is a trivial consequence of Lemma 2.2 of [1]. As for (16), let $G$ be a standard normal variable. Then

$$
\mathrm{E}\left\{\Psi\left(\frac{u}{\sigma_{g} \sqrt{X}}\right)\right\}=\frac{1}{2} \mathrm{P}\left\{G^{2} X>\frac{u^{2}}{\sigma_{g}^{2}}\right\} \asymp \frac{1}{2} \mathrm{E}\left\{|G|^{\alpha}\right\} \mathrm{P}\left\{X>\frac{u^{2}}{\sigma_{g}^{2}}\right\},
$$

the right-hand side following from a classic result of [5].

Since $\mathrm{E}\left\{|G|^{\alpha}\right\}=2^{\alpha / 2} \pi^{-1 / 2} \Gamma((1+\alpha) / 2)$ and $\mathrm{P}\{X>v\} \asymp C_{\alpha / 2} \sigma_{\alpha}^{\alpha / 2} v^{-\alpha / 2}$ (see Equations (3.7.2) and (1.2.8) of [8], respectively), the result now follows.

\section{Harmonisable fields}

The sub-Gaussian random fields of the previous section provide an interesting class of processes, in that they show that even a relatively minor perturbation of the Gaussian scenario leads to quite different behaviour of the excursion sets. Another, very rich, class of stable fields is given by the stationary, symmetric, $\alpha$-stable $(\mathrm{S} \alpha \mathrm{S})$ harmonisable fields. These are random fields possessing a spectral-type representation of the form

$$
f(t)=\operatorname{Re}\left\{\int_{\mathbb{R}^{N}} \mathrm{e}^{\mathrm{i} t \omega} Z(\mathrm{~d} \omega)\right\},
$$

where $Z$ is a complex, $\mathrm{S} \alpha \mathrm{S}$, Borel random measure on $\mathbb{R}^{N}$ with finite control measure $\mu$. (See [8, pp. 300-301] for details of this and the following representation.)

While (17) may explain from where the terminology comes, there is an alternative representation that will be much more useful for us, and which is given by

$$
f(t)=\left(C_{\alpha} b_{\alpha}^{-1} \mu_{0}\right)^{1 / \alpha} \sum_{k=1}^{\infty} \Gamma_{k}^{-1 / \alpha}\left(G_{k}^{(1)} \cos \left(t \omega_{k}\right)+G_{k}^{(2)} \sin \left(t \omega_{k}\right)\right),
$$

where $t \in \mathbb{R}^{N}$ and the product $t \omega_{k}$ is actually the inner product $\sum_{i=1}^{N} t(i) \omega_{k}(i)$. The $\left\{G_{k}^{(i)}\right\}, i=$ 1,2, are independent sequences of independent and identically distributed (i.i.d.) standard normal variables. Here $\left\{\Gamma_{k}\right\}$ is the sequence of arrival times of a unit rate Poisson process, $\left\{\omega_{k}\right\}$ is a sequence of i.i.d. $\mathbb{R}^{N}$-valued random variables with probability measure $\mu(\cdot) / \mu_{0}$, where

$$
\mu_{0}:=\mu\left(\mathbb{R}^{N}\right)
$$

and $\mu$ is the control measure for (17). The four sequences are independent of one another. The constants $C_{\alpha}$ and $b_{\alpha}$ are given by

$$
C_{\alpha}=\left(\int_{0}^{\infty} x^{-\alpha} \sin x \mathrm{~d} x\right)^{-1}= \begin{cases}(\Gamma(1-\alpha) \cos (\pi \alpha / 2))^{-1} & \text { if } \alpha \neq 1 \\ 2 / \pi & \text { if } \alpha=1\end{cases}
$$

and $b_{\alpha}=2^{\alpha / 2} \Gamma\left(1+\frac{1}{2} \alpha\right)$.

An important consequence of representation (18) is that if we condition on the sequences $\left\{\Gamma_{k}\right\}$ and $\left\{\omega_{k}\right\}$ then the conditioned field is stationary Gaussian. This will enable us, as in 
the sub-Gaussian case, to use conditional Gaussian arguments to prove the following result in which we establish the asymptotics of the expected Euler characteristic of the excursion sets of the real harmonisable stable fields. For simplicity, we restrict ourselves to the case of a compactly supported control measure $\mu$ with a bounded density, but we expect that the result remains true in greater generality.

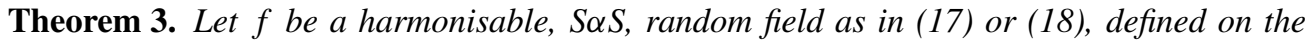
$N$-rectangle $T$ of (1). Assume that the control measure $\mu$ has compact support and a bounded density with respect to Lebesgue measure. Then

$$
\mathrm{E}\left\{\varphi\left(A_{u}(f, T)\right)\right\} \asymp u^{-\alpha} C_{\alpha} \mu_{0}\left(\frac{2^{1-\alpha / 2} \Gamma((1+\alpha) / 2)}{\sqrt{\pi} b_{\alpha}}+\frac{1}{2 \pi} \sum_{j=1}^{N} \mu_{j} T_{j}\right),
$$

where $\mu_{0}$ was defined in (19) and the $\mu_{j}, j=1, \ldots, N$, are the (normalized) moments

$$
\mu_{j}:=\mathrm{E}\left\{\left|\omega_{j}\right|\right\}=\int\left|\omega_{j}\right| \frac{\mu(\mathrm{d} \omega)}{\mu_{0}} .
$$

If, furthermore, $\mu$ is rotationally invariant (so that $f$ is isotropic) and $M$ is a compact convex domain, then

$$
\mathrm{E}\left\{\varphi\left(A_{u}(f, M)\right)\right\} \asymp u^{-\alpha} C_{\alpha} \mu_{0}\left(\frac{2^{1-\alpha / 2} \Gamma((1+\alpha) / 2)}{\sqrt{\pi} b_{\alpha}} \mathcal{L}_{0}(M)+\frac{\mu_{1}}{2 \pi} \mathcal{L}_{1}(M)\right),
$$

where $\mu_{1}$ is any of the (equivalent) moments given by (21).

Note, once again, how different this result is to the corresponding Gaussian one, and even to the sub-Gaussian one. Comparing, for example, (22) with its Gaussian counterpart (11) (take $j=0$ there) we see that while the leading term in the Gaussian case comes from the volume term $\mathcal{L}_{N}(M)$, in the harmonisable stable case it comes from the two lowest Lipschitz-Killing curvatures, $\mathscr{L}_{0}(M)$ and $\mathcal{L}_{1}(M)$.

To see why this should be the case, we shall postpone the rather technical proof of the theorem for a moment, take a moment to describe the principles involved, and then use them to obtain a heuristic proof of the theorem.

Consider representation (18) for harmonisable stable fields. From this, we can argue that, conditional on $f$ reaching a high level $u$, the first term, with the coefficient $\Gamma_{1}^{-1 / \alpha}$, will dominate not only all the other summands, but in fact their sum. (Formulating this properly, and then establishing it, is basically the main part of the technical proof.)

This being the case, $f$ will tend, at high levels, to look like a cosine function, with random height, frequency, and direction. Thus, for example, in $\mathbb{R}^{2}$, a typical high-level excursion set will look like that in the square of Figure 1, a sequence of strips looking like the tops of cosine waves, with small perturbations due to the terms in the representation other than the dominant one.

To count how many such strips there are, we need to look only at the boundary of the square. In fact, if we increase the size of the square, it is clear that the number of strips (and so the Euler characteristic) grows proportionately to the length of the edges, and not to the area. Thus, it should no longer be surprising that only $\mathcal{L}_{0}(M)$ and $\mathcal{L}_{1}(M)$ appear in $(22)$. (The $\mathcal{L}_{0}(M)$ term arises to 'catch' regions such as those in the lower-left corner of Figure 1.)

There is also another argument that will give us not only the results of Theorem 3, but even a little more. Again, it is only heuristic, but it is both elegant and simple, and since it may also 


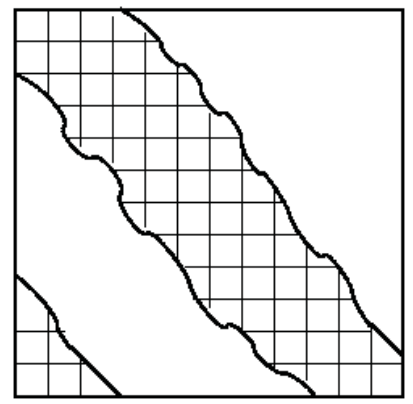

FIGURE 1: A 'typical' high-level excursion set for a harmonisable field on $[0,1]^{2}$.

be applicable to other problems, it is worth the space we shall devote to it. Its approach is via integral geometry.

We start with the assumption that it is only the first term of sum (18) that will be important, and so (ignoring multiplicative constants) look at the random field

$$
f(t)=\Gamma_{1}^{-1 / \alpha}\left(G_{1}^{(1)} \cos \left(t \omega_{1}\right)+G_{1}^{(2)} \sin \left(t \omega_{1}\right)\right)
$$

over a compact, convex $M$.

For a given direction $\omega \in \mathbb{R}^{N}$, let $P_{\omega}$ denote the projection onto the line containing the origin and $\omega$, and let

$$
M_{\omega}=\left\{P_{\omega} t: t \in M\right\}
$$

be the projection of $M$ onto this line. Conditioning on $\omega$, the Euler characteristic of the excursion set $A_{u}(f, M)$ is the same as the number of upcrossings of the level $u$ by the one-dimensional cosine wave on $M_{\omega}$, plus 1 if $f \geq u$ at the boundary point of $M_{\omega}$ closest to the origin.

If we now average over the Gaussian variables $G_{1}^{(1)}$ and $G_{1}^{(2)}$, then it is easy to check that, conditioned on $\omega$, the expected Euler characteristic should be proportional to

$$
\mathrm{P}\{f(0) \geq u\}+u^{-\alpha}\|\omega\|\left|T_{\omega}\right| \asymp u^{-\alpha}\left(k_{1}+k_{2}\|\omega\|\left|T_{\omega}\right|\right)
$$

for some constants $k_{1}$ and $k_{2}$ which we shall not worry about. Averaging over $\omega$ we find that

$$
\int_{\mathbb{R}^{N}}\left(k_{1}+k_{2}\|\omega\|\left|T_{\omega}\right|\right) \frac{\mu(\mathrm{d} \omega)}{\mu_{0}}=k_{1}+k_{2} \int_{\mathbb{R}^{N}}\left|T_{\omega}\right|\|\omega\| \frac{\mu(\mathrm{d} \omega)}{\mu_{0}} .
$$

Now assume that the measure $\mu$ is rotationally symmetric. Then the so-called projection theorem of integral geometry (cf. [7, Section 7.4]) gives us that the integral here is proportional to $\mathscr{L}_{1}(M)$. This gives $(22)$, once we compute the constants.

Indeed, we can go further than (22), dropping the isotropy assumption. In general, the quantity $\|\omega\|\left|M_{\omega}\right|$ can be expressed as

$$
\|\omega\|\left|M_{\omega}\right|=\sup _{t \in M}\langle\omega, t\rangle-\inf _{t \in M}\langle\omega, t\rangle=h_{M}(\omega)-h_{M}(-\omega),
$$

where

$$
h_{M}(\omega)=\sup _{t \in M}\langle\omega, t\rangle
$$


is the support function [7] of the convex body $M$. (Usually, the support function is considered as a function on the unit sphere $S\left(\mathbb{R}^{N}\right)$, but (24) is well defined as a function on $\mathbb{R}^{N}$.)

Following through with the constants, this argument would give, for compact convex bodies $M$,

$$
\begin{aligned}
& \mathrm{E}\left\{\varphi\left(A_{u}(f, M)\right)\right\} \\
& \quad \asymp u^{-\alpha} C_{\alpha} \mu_{0}\left(\frac{2^{1-\alpha / 2} \Gamma((1+\alpha) / 2)}{\sqrt{\pi} b_{\alpha}}+\frac{1}{2 \pi} \int_{\mathbb{R}^{N}}\left(h_{M}(\omega)-h_{M}(-\omega)\right) \frac{\mu(\mathrm{d} \omega)}{\mu_{0}}\right) .
\end{aligned}
$$

To see how this works for rectangles $T$, note that in this case the difference of the support functions appearing in (23) is just $\sum_{j=1}^{N}\left|\omega_{j}\right| T_{j}$. Substituting this into (25) gives us back (20).

We shall return to integral geometric arguments later, in Section 7, but for the moment we leave heuristics and geometric arguments and give the promised technical proof.

Proof of Theorem 3. We shall only give a proof of (20), the result for the rectangular parameter space $T$. The extension to compact, convex parameter spaces follows from Hadwiger's representation of additive functionals, as in the proof of Theorem 2.

The proof will follow the general lines of that of Theorem 2, in that it begins with a conditioning argument from which asymptotics are computed. The harmonisable case, however, is somewhat more complicated.

We begin with representation (18). If we condition on the sequences $\left\{\Gamma_{k}\right\}$ and $\left\{\omega_{k}\right\}$, it is immediate that the conditioned field is a stationary Gaussian field on $\mathbb{R}^{N}$ with mean 0 and covariance function

$$
R(t)=\gamma_{\alpha}^{2} \sum_{k=1}^{\infty} \Gamma_{k}^{-2 / \alpha} \cos \left(t \omega_{k}\right)
$$

where

$$
\gamma_{\alpha}:=\left(C_{\alpha} b_{\alpha}^{-1} \mu_{0}\right)^{1 / \alpha}
$$

Therefore, it has variance

$$
\widetilde{\sigma}^{2}=\gamma_{\alpha}^{2} \sum_{k=1}^{\infty} \Gamma_{k}^{-2 / \alpha}
$$

and second spectral moments

$$
\tilde{\lambda}_{i j}=\gamma_{\alpha}^{2} \sum_{k=1}^{\infty} \Gamma_{k}^{-2 / \alpha} \omega_{k}(i) \omega_{k}(j) .
$$

Our next step will be to apply the Gaussian theorem, Theorem 1, to the conditioned harmonisable process, and then use the fact that

$$
\mathrm{E}\left\{\varphi\left(A_{u}(f, T)\right\}=\mathrm{E}\left\{\mathrm{E}\left\{\varphi\left(A_{u}(f, T)\right) \mid \Gamma_{k}, \omega_{k}, k \geq 1\right\}\right\} .\right.
$$

As in the sub-Gaussian case, the iterated expectation requires justification, which will be provided only later in Lemma 5.

In order to apply the Gaussian result, we need first to verify three conditions: that the conditioned process is a.s. $C^{2}$, that the joint distributions of the various conditioned derivatives 
are nondegenerate, and that condition (9) on the moduli of continuity of the conditioned process is satisfied.

We tackle the first of these first, by showing that $f$, itself, is a.s. $C^{2}$. From this fact and Fubini's theorem, the same will be true of the conditioned processes.

Note that the assumption of compact support for the control measure $\mu$ implies, by Corollary 11.7.5 of [8], that $f$ is absolutely continuous. Write $f_{i}$ and $f_{i j}$ for the various first- and secondorder partial derivatives of $f$. Exploiting representation (17), it is easy to check that as versions of its partial derivatives we can take the random fields

$$
f_{j}(t)=\operatorname{Re}\left\{\int_{\mathbb{R}^{N}} \mathrm{e}^{\mathrm{i} t \omega} \mathrm{i} \omega(j) Z(\mathrm{~d} \omega)\right\} .
$$

Since these are, again, harmonisable fields with compactly supported control measures, they are all continuous. From this, it follows that $f$ is $C^{1}$. Applying the same argument to the derivatives of the $f_{j}$ gives that $f$ is $C^{2}$.

We now turn to the issue of the nondegeneracy of the joint distribution of the various derivatives. Applying representation (18), we have

$$
f_{i}(t)=\left(C_{\alpha} b_{\alpha}^{-1} \mu_{0}\right)^{1 / \alpha} \sum_{k=1}^{\infty} \Gamma_{k}^{-1 / \alpha} \omega_{k}(i)\left(-G_{k}^{(1)} \sin \left(t \omega_{k}\right)+G_{k}^{(2)} \cos \left(t \omega_{k}\right)\right)
$$

and

$$
f_{i j}(t)=-\left(C_{\alpha} b_{\alpha}^{-1} \mu_{0}\right)^{1 / \alpha} \sum_{k=1}^{\infty} \Gamma_{k}^{-1 / \alpha} \omega_{k}(i) \omega_{k}(j)\left(G_{k}^{(1)} \sin \left(t \omega_{k}\right)+G_{k}^{(2)} \cos \left(t \omega_{k}\right)\right)
$$

for $i, j=1, \ldots, N$. In particular, the joint distribution of $f$ and its partial derivatives at time 0 (up to a positive multiplicative constant) is that of the random vector

$$
\left\{\sum_{k=1}^{\infty} \Gamma_{k}^{-1 / \alpha} G_{k}^{(1)}, \sum_{k=1}^{\infty} \Gamma_{k}^{-1 / \alpha} \omega_{k}(i) G_{k}^{(2)}, \sum_{k=1}^{\infty} \Gamma_{k}^{-1 / \alpha} \omega_{k}(i) \omega_{k}(j) G_{k}^{(1)}\right\}_{i, j=1}^{N} .
$$

Since we have assumed that the distribution of the random vectors $\omega_{k}$ has a nonvanishing absolutely continuous component, this is, with probability 1, a nondegenerate Gaussian vector.

It remains to check that condition (9) on the moduli of continuity of the second-order derivatives of the conditioned process is satisfied. By Corollary 11.3.2 of [2], writing $C_{f_{i j}}(t)$ for the covariance function of the second-order partial derivatives $f_{i j}$, this condition will be satisfied if, for small enough $t$, and some finite $K, \eta>0$,

$$
\max _{i, j}\left|C_{f_{i j}}(0)-C_{f_{i j}}(t)\right| \leq K|\ln | t||^{-(1+\eta)} .
$$

However, using representation (28) for the $f_{i j}$, it is easy to compute an expression for the $C_{f_{i j}}$ and, using the compact support of the $\omega_{k}(i)$, to see from this that (29) is not only satisfied, but that the bound on the right-hand side can be taken of order $|t|^{2}$.

With all the conditions checked, we can now apply Theorem 1 to the conditioned harmonisable process, from which it follows that

$$
\mathrm{E}\left\{\varphi\left(A_{u}(f, T)\right)\right\}=\mathrm{E}\left\{\mathrm{e}^{-u^{2} / 2 \widetilde{\sigma}^{2}} \sum_{n=1}^{N} \sum_{J \in \mathcal{O}_{n}} \frac{|J|\left|\widetilde{\Lambda}_{J}\right|^{1 / 2}}{(2 \pi)^{(n+1) / 2} \widetilde{\sigma}^{n}} H_{n-1}\left(\frac{u}{\widetilde{\sigma}}\right)+\Psi\left(\frac{u}{\widetilde{\sigma}}\right)\right\},
$$

where the expectation on the right-hand side is over the $\Gamma_{k}$ and $\omega_{k}$, and, for $J \in \mathcal{O}_{n}$, the $n \times n$ matrix $\widetilde{\Lambda}_{J}$ bears the same relation to the $\widetilde{\lambda}_{i j}$ that $\Lambda_{J}$ does to the $\lambda_{i j}$. 
The term $\mathrm{E}\{\Psi(u / \widetilde{\sigma})\}$ can be handled much as in the proof of Theorem 2, using (16) to show that

$$
\mathrm{E}\left\{\Psi\left(\frac{u}{\widetilde{\sigma}}\right)\right\} \asymp u^{-\alpha} \frac{2^{1-\alpha / 2} C_{\alpha} \mu_{0} \Gamma((1+\alpha) / 2)}{\sqrt{\pi} b_{\alpha}} .
$$

As for the other terms, it is clear, expanding the Hermite polynomials, that we need to study the asymptotics of terms of the form

$$
\mathrm{E}\left\{\frac{\left|\widetilde{\Lambda}_{J}\right|^{1 / 2} u^{n-1-2 j}}{\widetilde{\sigma}^{2 n-1-2 j}} \mathrm{e}^{-u^{2} / 2 \widetilde{\sigma}^{2}}\right\}
$$

for $0 \leq j \leq\lfloor(n-1) / 2\rfloor$.

To do this, we need a little notation. For $k \geq 1$ and a facet $J$ of dimension $n$, define the $n \times n$ matrices $W_{k} \equiv W_{k}(J)$ by setting

$$
W_{k}(i, j)=\omega_{k}(i) \omega_{k}(j), \quad i, j \in \sigma(J) .
$$

Then (31) can be rewritten as

$$
\gamma_{\alpha}^{1 / 2+j} \mathrm{E}\left\{\frac{\left|\operatorname{det}\left(\sum_{1}^{\infty} \Gamma_{k}^{-2 / \alpha} W_{k}\right)\right|^{1 / 2} u^{n-1-2 j}}{\left(\sum_{1}^{\infty} \Gamma_{k}^{-2 / \alpha}\right)^{(2 n-1-2 j) / 2}} \exp \left(\frac{-u^{2}}{2 \gamma_{\alpha}^{2} \sum_{1}^{\infty} \Gamma_{k}^{-2 / \alpha}}\right)\right\} .
$$

Our first step in handling this expectation will be to truncate the sum in the numerator. Note that, for any two $n \times n$ matrices $A$ and $B$, the standard expansion of a determinant shows that

$$
\operatorname{det}(A+B) \leq \operatorname{det}(A)+\sum_{m=1}^{n} C_{n m}\|A\|^{n-m}\|B\|^{m}
$$

for some combinatorial constants $C_{n m}$ that we allow to change from line to line and $\|A\|=$ $\max _{i j}\left|a_{i j}\right|$. Furthermore, since $\sqrt{x+y} \leq \sqrt{x}+\sqrt{y}$, we can apply this to the numerator of (33) to see that there is a constant $C$ such that

$$
\begin{aligned}
& \left|\left(\operatorname{det}\left(\sum_{1}^{\infty} \Gamma_{k}^{-2 / \alpha} W_{k}\right)\right)^{1 / 2}-\left(\operatorname{det}\left(\Gamma_{1}^{-2 / \alpha} W_{1}\right)\right)^{1 / 2}\right| \\
& \quad \leq C \sum_{m=1}^{n}\left(\Gamma_{1}^{-2 / \alpha}\left\|W_{1}\right\|\right)^{(n-m) / 2}\left(\sum_{k=2}^{\infty} \Gamma_{k}^{-2 / \alpha}\left\|W_{k}\right\|\right)^{m / 2} .
\end{aligned}
$$

We now claim that, in view of the above inequality, the expectation in (33) differs from

$$
\mathrm{E}\left\{\frac{\left|\operatorname{det}\left(\Gamma_{1}^{-2 / \alpha} W_{1}\right)\right|^{1 / 2} u^{n-1-2 j}}{\left(\sum_{1}^{\infty} \Gamma_{k}^{-2 / \alpha}\right)^{(2 n-1-2 j) / 2}} \exp \left(\frac{-u^{2}}{2 \gamma_{\alpha}^{2} \sum_{1}^{\infty} \Gamma_{k}^{-2 / \alpha}}\right)\right\}
$$

by no more than a factor of $o\left(u^{-\alpha}\right)$. Note that in the simplest case, when $N=n=1$ and $j=0$, this is precisely Lemma 2.4 of [3]. To prove the general claim we need here, observe that, by (34) and the assumption of the bounded support of the random vectors $\omega_{k}$, it is enough to prove that, for every $0 \leq j \leq\lfloor(n-1) / 2\rfloor$ and $1 \leq m \leq n$,

$$
u^{n-1-2 j} \mathrm{E}\left\{\frac{\Gamma_{1}^{-(n-m) / \alpha}\left(\sum_{k=2}^{\infty} \Gamma_{k}^{-2 / \alpha}\right)^{m / 2}}{\left(\sum_{1}^{\infty} \Gamma_{k}^{-2 / \alpha}\right)^{(2 n-1-2 j) / 2}} \exp \left(\frac{-u^{2}}{2 \gamma_{\alpha}^{2} \sum_{1}^{\infty} \Gamma_{k}^{-2 / \alpha}}\right)\right\}=o\left(u^{-\alpha}\right) \quad \text { as } u \rightarrow \infty .
$$


To this end, note that the left-hand side of (36) can be bounded by

$$
u^{n-1-2 j} \mathrm{E}\left\{\frac{\left(\sum_{k=2}^{\infty} \Gamma_{k}^{-2 / \alpha}\right)^{1 / 2}}{\left(\sum_{1}^{\infty} \Gamma_{k}^{-2 / \alpha}\right)^{(n-2 j) / 2}} \exp \left(\frac{-u^{2}}{2 \gamma_{\alpha}^{2} \sum_{1}^{\infty} \Gamma_{k}^{-2 / \alpha}}\right)\right\} .
$$

Take a small $\varepsilon>0$ and write (37) as a sum of two terms, the first when the expectation is restricted to the event $\left\{\left(\sum_{k=2}^{\infty} \Gamma_{k}^{-2 / \alpha}\right)^{1 / 2}>\varepsilon u\right\}$, and the second arising when the expectation is restricted to the complementary event.

Noting that, for any $\theta>0$, there is a finite $c$ such that $\sigma^{-2 \theta} \exp \left\{-u^{2} / \sigma^{2}\right\} \leq c u^{-2 \theta}$ for all $u, \sigma>0$, we see that the first term can be bounded by

$$
c u^{-1} \mathrm{E}\left\{\left(\sum_{k=2}^{\infty} \Gamma_{k}^{-2 / \alpha}\right)^{1 / 2} \mathbf{1}_{\left\{\left(\sum_{k=2}^{\infty} \Gamma_{k}^{-2 / \alpha}\right)^{1 / 2}>\varepsilon u\right\}}\right\} .
$$

Since $\mathrm{P}\left(\sum_{k=2}^{\infty} \Gamma_{k}^{-2 / \alpha}>u\right)=o\left(u^{-\alpha / 2}\right)$ (see [8, p. 27]), it follows that the first term in (37) is $o\left(u^{-\alpha}\right)$ as $u \rightarrow \infty$ for every fixed $\varepsilon>0$. On the other hand, the second term is, clearly, bounded by

$$
\varepsilon u^{n-2 j} \mathrm{E}\left\{\frac{1}{\left(\sum_{1}^{\infty} \Gamma_{k}^{-2 / \alpha}\right)^{(n-2 j) / 2}} \exp \left(\frac{-u^{2}}{2 \gamma_{\alpha}^{2} \sum_{1}^{\infty} \Gamma_{k}^{-2 / \alpha}}\right)\right\} .
$$

Since the sum $\sum_{1}^{\infty} \Gamma_{k}^{-2 / \alpha}$ is a positive $\alpha / 2$-random variable, it has a density of asymptotic order $x^{-(1+\alpha / 2)}$, and straightforward estimates now show that the expectation above is of order $u^{-(n-2 j+\alpha)}$ as $u \rightarrow \infty$. Consequently,

$$
\limsup _{u \rightarrow \infty} u^{\alpha}(\text { the second term in }(37)) \leq c \varepsilon
$$

for some $c>0$, and letting $\varepsilon \rightarrow 0$ proves (36).

Returning now to (35), which is what is left to study, we note that it has a simple structure, since it is immediate from the definition of $W_{1}=W_{1}(J)$ that it is a rank 1 matrix for all $J \in \mathcal{O}_{n}$. Hence,

$$
\operatorname{det}\left(\Gamma_{1}^{-2 / \alpha} W_{1}\right)=\Gamma_{1}^{-n / \alpha} \operatorname{det}\left(W_{1}\right) \equiv 0,
$$

unless $n=1$. Thus, taking $n=1$ and $j=0$ (now the only possible value of $j$ ) in (35), recalling the independence of the $\omega_{k}$ and $\Gamma_{k}$, we need only consider $N$ terms of the form

$$
\mathrm{E}\left\{\left|\omega_{1}(i)\right|\right\} \mathrm{E}\left\{\frac{\Gamma_{1}^{-1 / \alpha}}{\left(\sum_{1}^{\infty} \Gamma_{k}^{-2 / \alpha}\right)^{1 / 2}} \exp \left(\frac{-u^{2}}{2 \gamma_{\alpha}^{2} \sum_{1}^{\infty} \Gamma_{k}^{-2 / \alpha}}\right)\right\}, \quad i=1, \ldots, N .
$$

The first expectation here is, by definition, $\mu_{i}$, while the second converges to the constant $\mu_{0} C_{\alpha}$ by the first part of Theorem 2.2 of [3]. Putting everything together proves (20).

\section{Concatenated-harmonisable fields}

For our final class of examples, we shall introduce a class of random fields which, to the best of the authors' knowledge, have not been studied earlier. We actually discovered them by looking for a class of examples which 'interpolated' between the sub-Gaussian ones, for which all the $\mathcal{L}_{j}$ appear in the asymptotic formula for the mean Euler characteristic of excursion sets, and the harmonisable ones, for which only $\mathscr{L}_{0}$ and $\mathcal{L}_{1}$ appear. However, having found them for 
this rather artificial purpose, we believe that they actually present an interesting class of stable fields that will provide useful models in applied settings.

To define this new class of random fields, we take the representation

$$
f(t)=\left(\frac{C_{\alpha} \mu_{0}}{b_{\alpha}}\right)^{1 / \alpha} \sum_{k=1}^{\infty} \Gamma_{k}^{-1 / \alpha} \sum_{\ell=1}^{N^{\prime}}\left(G_{k \ell}^{(1)} \cos \left(t \omega_{k \ell}\right)+G_{k \ell}^{(2)} \sin \left(t \omega_{k \ell}\right)\right)
$$

where the $\Gamma_{k}$ are as in (18), the $\left\{G_{k \ell}^{(i)}\right\}, i=1,2, \ell=1, \ldots, N^{\prime}$, are independent, standard Gaussian random variables, and the $\left\{\omega_{k \ell}\right\}$ are independent with the distribution of the $\omega_{k}$ of (18). The parameter $N^{\prime}$ satisfies $1 \leq N^{\prime} \leq N$. (When $N^{\prime}=1$, we recover the harmonisable fields of (18).) We call such fields concatenated-harmonisable, the adjective 'concatenated' coming from the innermost sum in (38).

If the heuristics used before work again, the dominant term in the expansion,

$$
\left(\frac{C_{\alpha} \mu_{0}}{b_{\alpha}}\right)^{1 / \alpha} \Gamma_{1}^{-1 / \alpha} \sum_{\ell=1}^{N^{\prime}}\left(G_{k \ell}^{(1)} \cos \left(t \omega_{k \ell}\right)+G_{k \ell}^{(2)} \sin \left(t \omega_{k \ell}\right)\right)
$$

should determine the properties of the high-level excursion sets. This random field is quite different from that of the simple random wave generated by the first term of the harmonisable processes, and so the arguments there, based on examples as in Figure 1, are not going to carry over easily to the current situation. We did find an integral geometric argument which justified Theorem 4, below, but it was no longer simple and, since it was also nonrigorous, we shall not use it here.

In order to state the result, we need a little more notation. Changing slightly that of the proof of Theorem 3, choose a facet $J \in \mathcal{O}_{n}$, and, for each $k \geq 1$, define the $n \times n$ matrix $W_{k}(J)$ with elements

$$
\left(W_{k}(J)\right)_{i j}=\sum_{\ell=1}^{N^{\prime}} \omega_{k \ell}(i) \omega_{k \ell}(j)
$$

for $i, j \in \sigma(J)$. Furthermore, define the ( $k$-independent) parameters

$$
\Lambda(J):=\mathrm{E}\left\{\left|\operatorname{det}\left(W_{k}(J)\right)\right|^{1 / 2}\right\} .
$$

Theorem 4. Let $f$ be a concatenated-harmonisable, $S \alpha S$ random field as in (38), defined on the $N$-rectangle $T$ of (1). Assume that the control measure $\mu$ has compact support, and a bounded density with respect to the Lebesgue measure. Then

$$
\mathrm{E}\left\{\varphi\left(A_{u}(f, T)\right)\right\} \asymp u^{-\alpha} \mu_{0} C_{\alpha}\left(K_{0}+\sum_{n=1}^{N^{\prime}} K_{n} \sum_{J \in \mathcal{O}_{n}}|J| \Lambda(J)\right),
$$

where

$$
K_{0}=\frac{2^{1-\alpha / 2} \Gamma((1+\alpha) / 2)\left(N^{\prime}\right)^{\alpha / 2}}{\sqrt{\pi} b_{\alpha}},
$$

and, for $n=1, \ldots, N^{\prime}, K_{n}$ is given by (43), below. 
If, furthermore, $\mu$ is rotationally invariant (so that is isotropic) and $M$ is a compact convex domain, then

$$
\mathrm{E}\left\{\varphi\left(A_{u}(f, M)\right)\right\} \asymp u^{-\alpha} \mu_{0} C_{\alpha} \sum_{n=0}^{N^{\prime}} K_{n} \Lambda_{n} \mathcal{L}_{n}(M),
$$

where $\Lambda_{n}$ is given by (40) for any $n$-dimensional facet $J$.

Proof. We shall prove only (41), with result (42) for isotropic processes on convex parameter sets following from the usual integral geometric argument via Hadwiger's theorem.

The proof follows the lines of that of Theorem 3, and so we start by conditioning on the sequences $\left\{\Gamma_{k}\right\}$ and $\left\{\omega_{k \ell}\right\}$, to obtain a stationary Gaussian process on $\mathbb{R}^{N}$ with mean 0 , variance

$$
\widetilde{\sigma}^{2}=\gamma_{\alpha}^{2} N^{\prime} \sum_{k=1}^{\infty} \Gamma_{k}^{-2 / \alpha}
$$

where $\gamma_{\alpha}$ is as in (26), and second-order spectral moments

$$
\tilde{\lambda}_{i j}=\gamma_{\alpha}^{2} \sum_{k=1}^{\infty} \Gamma_{k}^{-2 / \alpha} \sum_{\ell=1}^{N^{\prime}} \omega_{k \ell}(i) \omega_{k \ell}(j) .
$$

We need to prove that the iterated expectation argument for computing $\mathrm{E}\left\{\varphi\left(A_{u}(f, T)\right)\right\}$ is valid. This is done in Lemma 5, below. The arguments that worked in the harmonisable case also work here to show that the conditionally Gaussian process satisfies all the conditions of Theorem 1. Thus, our task becomes one of evaluating (30) once again, albeit with the new definitions of the variables there. The term involving $\Psi$ changes from that in the previous proof only insofar as there is now an additional factor of $N^{\prime}$ in the definition of $\widetilde{\sigma}^{2}$ in (27) and this gives the first term in (41) (cf. Lemma 1).

As far as the other terms are concerned, the argument is identical to that in the proof of Theorem 3 as far as (35), and so what remains to compute is

$$
\left(N^{\prime}\right)^{-(2 n-1-2 j) / 2} \mathrm{E}\left\{\frac{\left|\operatorname{det}\left(\Gamma_{1}^{-2 / \alpha} W_{1}\right)\right|^{1 / 2} u^{n-1-2 j}}{\left(\sum_{1}^{\infty} \Gamma_{k}^{-2 / \alpha}\right)^{(2 n-1-2 j) / 2}} \exp \left(\frac{-u^{2}}{2 \gamma_{\alpha}^{2} N^{\prime} \sum_{1}^{\infty} \Gamma_{k}^{-2 / \alpha}}\right)\right\}
$$

for $1 \leq n \leq N^{\prime}$ and $0 \leq j \leq\lfloor(n-1) / 2\rfloor$. The restrictions on $n$ follow from the fact that the matrices $W_{k}(J)$ have rank not exceeding $N^{\prime}$ and, since the $\omega_{k \ell}$ have a distribution with a nonvanishing absolutely continuous component, this rank will equal $\min \left(n, N^{\prime}\right)$, with positive probability.

Since $W_{1}$ is independent of the $\Gamma_{k}$, we can rewrite the above as

$$
\begin{aligned}
& \left(N^{\prime}\right)^{-(2 n-1-2 j) / 2} \mathrm{E}\left\{\left|\operatorname{det}\left(W_{1}(J)\right)\right|^{1 / 2}\right\} \\
& \times \mathrm{E}\left\{\frac{\Gamma_{1}^{-n / \alpha} u^{n-1-2 j}}{\left(\sum_{1}^{\infty} \Gamma_{k}^{-2 / \alpha}\right)^{(2 n-1-2 j) / 2}} \exp \left(\frac{-u^{2}}{2 \gamma_{\alpha}^{2} N^{\prime} \sum_{1}^{\infty} \Gamma_{k}^{-2 / \alpha}}\right)\right\} .
\end{aligned}
$$

The first expectation here is, by definition, $\Lambda(J)$. As for the second, its asymptotics follow from Lemma 2, below, to give

$$
u^{n-1-2 j} \mu_{0} C_{\alpha} C_{n j} u^{-(\alpha+(n-1-2 j))}=\mu_{0} C_{\alpha} C_{n j} u^{-\alpha},
$$


where

$$
C_{n j}:=\alpha C_{\alpha / 2} \sigma_{\alpha}^{\alpha / 2} b_{\alpha}^{-1} 2^{(\alpha+n-2 j-3) / 2} \Gamma\left(\frac{\alpha+n-1-2 j}{2}\right) \gamma_{\alpha}^{n-1-2 j}\left(N^{\prime}\right)^{(\alpha-n) / 2} .
$$

If we now substitute this back into (30), collect all the constants appearing in the Hermite polynomials, and define

$$
K_{n}=\frac{(n-1) !}{(2 \pi)^{(n+1) / 2}} \sum_{j=0}^{\lfloor(n-1) / 2\rfloor} \frac{(-1)^{j} C_{n j}}{j !(n-1-2 j) ! 2^{j}},
$$

a few lines of algebra yield (41), and we are done.

Lemma 2. Maintaining the above notation, set

$$
X=\sum_{j=1}^{\infty} \Gamma_{j}^{-2 / \alpha}, \quad n \geq 0, \beta>n / 2,
$$

and take $\gamma>0$. Then

$$
\begin{aligned}
& \mathrm{E}\left\{\Gamma_{1}^{-n / \alpha} X^{-\beta} \mathrm{e}^{-u^{2} / 2 \gamma^{2} X}\right\} \\
& \quad \asymp \alpha 2^{(\alpha+2 \beta-n-2) / 2} C_{\alpha / 2} \sigma_{\alpha}^{\alpha / 2} \gamma^{\alpha+2 \beta-n} \Gamma\left(\beta+\frac{1}{2}(\alpha-n)\right) u^{-(\alpha+2 \beta-n)} .
\end{aligned}
$$

Proof. The proof will proceed by establishing asymptotic upper and lower bounds for the expectation, which we shall denote by $I_{u}$.

For the upper bound, note that, since $\Gamma_{1}^{-2 / \alpha} \leq X$, it is immediate that

$$
I_{u} \leq \mathrm{E}\left\{X^{-(\beta-n / 2)} \mathrm{e}^{-u^{2} / 2 \gamma^{2} X}\right\} .
$$

However, the asymptotics of the right-hand side are covered by Lemma 1, and so we find that the right-hand side of (44) provides an upper bound for the asymptotics of $I_{u}$.

For the lower bound, fix $\varepsilon>0$ and again exploit the fact that $\Gamma_{1}^{-2 / \alpha} \leq X$ to see that

$$
\begin{aligned}
I_{u} & \geq \mathrm{E}\left\{\Gamma_{1}^{-n / \alpha} X^{-\beta} \mathrm{e}^{\left.-u^{2} / 2 \gamma^{2} \Gamma_{1}^{-2 / \alpha}\right\}}\right. \\
& \geq(1+\epsilon)^{-\beta} \mathrm{E}\left\{\Gamma_{1}^{-n / \alpha}\left(\Gamma_{1}^{-2 / \alpha}\right)^{-\beta} \mathrm{e}^{-u^{2} / 2 \gamma^{2} \Gamma_{1}^{-2 / \alpha}} \mathbf{1}_{\left\{\sum_{j=2}^{\infty} \Gamma_{j}^{-2 / \alpha} \leq \varepsilon \Gamma_{1}^{-2 / \alpha}\right\}}\right\} \\
& \geq(1+\epsilon)^{-\beta} \mathrm{E}\left\{\Gamma_{1}^{-n / \alpha}\left(\Gamma_{1}^{-2 / \alpha}\right)^{-\beta} \mathrm{e}^{-u^{2} / 2 \gamma^{2} \Gamma_{1}^{-2 / \alpha}} \mathbf{1}_{\left\{Y \leq \varepsilon \Gamma_{1}^{-2 / \alpha}\right\}}\right\},
\end{aligned}
$$

where $Y$ is a copy of $X$ independent of $\Gamma_{1}$. Now fix $M>0$, and note that

$$
\begin{aligned}
I_{u} & \geq(1+\varepsilon)^{-\beta} \mathrm{P}\{X \leq M\} \mathrm{E}\left\{\Gamma_{1}^{-n / \alpha}\left(\Gamma_{1}^{-2 / \alpha}\right)^{-\beta} \mathrm{e}^{-u^{2} / 2 \gamma^{2} \Gamma_{1}^{-2 / \alpha}} \mathbf{1}_{\left\{\Gamma_{1}^{-2 / \alpha} \geq M / \varepsilon\right\}}\right\} \\
& =(1+\varepsilon)^{-\beta} \mathrm{P}\{X \leq M\} \mathrm{E}\left\{\left(\Gamma_{1}^{-2 / \alpha}\right)^{-(\beta-n / 2)} \mathrm{e}^{-u^{2} / 2 \gamma^{2} \Gamma_{1}^{-2 / \alpha}} \mathbf{1}_{\left\{\Gamma_{1}^{-2 / \alpha} \geq M / \varepsilon\right\}}\right\} .
\end{aligned}
$$

The expectation here is similar to that in (45) and Lemma 1, with $\Gamma_{1}^{-2 / \alpha}$ replacing $X$. However, these two random variables have precisely the same tail behaviour, and a check of the proof of Lemma 1 (cf. Lemma 2.2 of [1]) shows that this is all that entered into the asymptotic behaviour of the expectation. Hence,

$$
I_{u} \gtrsim(1+\varepsilon)^{-\beta} \mathrm{P}\{X \leq M\} \mathrm{E}\left\{X^{-(\beta+n / 2)} \mathrm{e}^{-u^{2} / 2 \gamma^{2} X}\right\} .
$$

Sending $\varepsilon \rightarrow 0$ and $M \rightarrow \infty$ completes the proof. 


\section{On the mean Lipschitz-Killing curvatures of excursion sets}

Throughout this paper, we have concentrated on the expected Euler characteristics of excursion sets. However, at least in the isotropic cases, these results are immediately extendable to expected Lipschitz-Killing curvatures, via a result known as Crofton's formula.

To state Crofton's formula, we start with the affine $\operatorname{Grassmanian} \operatorname{Graff}(N, k)$ of all $k$-dimensional flats in $\mathbb{R}^{N}$, viz. of all $k$-dimensional linear subspaces of $\mathbb{R}^{N}$ not necessarily passing through the origin. On $\operatorname{Graff}(N, k)$ there is a natural Haar measure, known as the kinematic measure, which we denote by $\lambda_{k}^{N}$. Its precise definition and normalization will not be important to us. Crofton's formula states that if $M$ is a locally convex, sufficiently smooth, stratified manifold, then

$$
\int_{\operatorname{Graff}(N, N-k)} \mathcal{L}_{j}(M \cap V) \mathrm{d} \lambda_{N-k}^{N}(V)=\left[\begin{array}{c}
k+j \\
j
\end{array}\right] \mathcal{L}_{k+j}(M) .
$$

Now let $f$ be one of the stable random fields of this paper, and let $M$ be a compact, convex set in $\mathbb{R}^{N}$. If $f$ is isotropic then, recalling that the Euler functional $\varphi$ is also $\mathscr{L}_{0}$, all of our results can be written in the form

$$
\mathrm{E}\left\{\mathcal{L}_{0}\left(A_{u}(f, M)\right)\right\}=\sum_{k=0}^{N} C_{k}(u) \mathcal{L}_{k}(M)
$$

for some functions $C_{k}(u)$. The $C_{k}(u)$ depend on the parameters of $f$ and, quite often, are identically 0 . What is important, however, is that they are dependent neither on $M$ nor on $N=\operatorname{dim}(M)$.

If $M$ is compact and convex so is $M \cap V$ for any $V \in \operatorname{Graff}(N, k)$. Thus, exploiting Crofton's formula twice, and (47) once, we have

$$
\begin{aligned}
\mathrm{E}\left\{\mathcal{L}_{j}\left(A_{u}(f, M)\right)\right\} & =\mathrm{E}\left\{\int_{\operatorname{Graff}(N, N-j)} \mathcal{L}_{0}\left(A_{u}(f, M) \cap V\right) \mathrm{d} \lambda_{N-j}^{N}(V)\right\} \\
& =\int_{\operatorname{Graff}(N, N-j)} \mathrm{E}\left\{\mathcal{L}_{0}\left(A_{u}(f, M) \cap V\right)\right\} \mathrm{d} \lambda_{N-j}^{N}(V) \\
& =\int_{\operatorname{Graff}(N, N-j)} \sum_{k=0}^{N} C_{k}(u) \mathcal{L}_{k}(M \cap V) \mathrm{d} \lambda_{N-j}^{N}(V) \\
& =\sum_{k=0}^{N} C_{k}(u) \int_{\operatorname{Graff}(N, N-j)} \mathcal{L}_{k}(M \cap V) \mathrm{d} \lambda_{N-j}^{N}(V) \\
& =\sum_{k=0}^{N-j}\left[\begin{array}{c}
j+k \\
k
\end{array}\right] C_{k}(u) \mathcal{L}_{j+k}(M),
\end{aligned}
$$

the change on the range of summation coming from the fact that $\mathcal{L}_{l}(M) \equiv 0$ for all $l>N$.

Using this, all the formulae in this paper for the asymptotics of the mean Euler characteristics, in the isotropic cases, can be extended to asymptotics for the mean Lipschitz-Killing curvatures.

Of course, something has to be said about justifying the change of order of integration and expectation in (48), which we shall do in Lemma 6, below. 


\section{Appendix A. On the finiteness of expectations}

A serious technical point which we avoided throughout the paper was justifying the conditional expectation arguments

$$
\mathrm{E}\left\{\varphi\left(A_{u}(f, T)\right)\right\}=\mathrm{E}\left\{\mathrm{E}\left\{\varphi\left(A_{u}(f, T)\right) \mid \mathcal{F}\right\}\right\},
$$

where $f$ was stable and $\mathcal{F}$ was the information we assumed to make $f$ conditionally Gaussian.

The need for justification lies in the fact that Euler characteristics need not be positive. (Were they positive, (49) would always hold, with both sides being finite or infinite together.) We shall show that

$$
\mathrm{E}\left\{\left|\varphi\left(A_{u}(f, T)\right)\right|\right\}<\infty,
$$

which is sufficient for (49), restricting ourselves to rectangular parameter spaces only. The same arguments can also be applied for general compact domains, the only difference being a heavier investment in notation.

Before starting the proof of (50), we note that all the random fields that we considered in this paper are suitably regular Morse functions, in the terminology of Chapter 6 of [2]. This follows from a Fubini argument and the fact that, with probability 1, the conditionally Gaussian random fields on which we based all our calculations are of this kind. This, in turn, follows from the fact that they all satisfy the conditions of Theorem 1, which, by Corollary 11.3.2 of [2], implies that they are suitably regular Morse functions.

Turning now to the proof of (50), let $J$, as usual, be an $n$-dimensional facet of an $N$-dimensional rectangle $T$. Then, for a Morse function, the Euler characteristic of the excursion set $A_{u}(f, T)$ can be represented as an alternating sum, over all $J$, of the numbers of critical points of various indices of $f_{\mid J}$ over $A_{u}\left(f_{\mid J}, J\right)$ (cf. Section 9.4 of [2]). This sum is trivially no greater, in absolute value, than the total number, over all $J$, of the number of critical points of $f_{\mid J}$ over $J$. Thus, in order to establish (50), it will suffice to show that, for every facet $J$,

$$
\mathrm{E}\{\mathrm{E}\{N(f, J) \mid \mathcal{F}\}\}<\infty,
$$

where $N(f, J)$ is the number of critical points of $f$ when restricted to $J$.

In order to compute the inner expectation, recall that $f$ is conditionally a stationary Gaussian random field in all the cases of interest to us. Call this process $\hat{f}$. Then it follows immediately from Theorem 11.2.1 of [2] and the independence of first- and second-order partial derivatives of Gaussian fields (cf. [2] Section 11.7) that

$$
\mathrm{E}\{N(\hat{f}, J) \mid \mathcal{F}\}=\frac{\mathrm{E}\left\{\left|\operatorname{det} \nabla^{2} \hat{f}_{|J|}\right|\right\}}{\operatorname{det} \Sigma_{\hat{f}_{\mid J}}},
$$

where $\nabla^{2} \hat{f}_{\mid J}$ is the $n \times n$ matrix of second-order derivatives of $\hat{f}_{\mid J}$ and $\Sigma_{\hat{f}_{\mid J}}$ is the $n \times n$ covariance matrix of its first-order derivatives.

With these preliminaries behind us, we can begin establishing the two main results of this appendix, Lemmas 3 and 5.

Lemma 3. Let $f$ be a sub-Gaussian process satisfying the conditions of Theorem 2. Then, for every $u \in \mathbb{R}, \mathrm{E}\left\{\left|\varphi\left(A_{u}(f, T)\right)\right|\right\}$ is finite. 
Proof. Recall that in the sub-Gaussian case $f$ is no more that $X^{1 / 2} g$, where $g$ is Gaussian and $X$ is positive $\alpha / 2$-stable. Thus, given $X=x$, the conditioned process $\hat{f}$ of (51) is no more that $x^{1 / 2} g$. It therefore follows that, for a facet $J$ of dimension $n$,

$$
\begin{aligned}
\mathrm{E}\{N(f, J)\} & =\mathrm{E}\{\mathrm{E}\{N(\hat{f}, J) \mid X\}\} \\
& =\mathrm{E}\left\{\frac{\mathrm{E}\left\{\left|\operatorname{det} \nabla^{2} \hat{f}_{\mid J}\right|\right\}}{\operatorname{det} \Sigma_{\hat{f}_{\mid J}}} \mid X\right\} \\
& =\mathrm{E}\left\{\frac{X^{n / 2} \mathrm{E}\left\{\left|\operatorname{det} \nabla^{2} g_{\mid J}\right|\right\}}{X^{n / 2} \operatorname{det} \Sigma_{g_{\mid J}}} \mid X\right\} \\
& =\frac{\mathrm{E}\left\{\left|\operatorname{det} \nabla^{2} g_{\mid J}\right|\right\}}{\operatorname{det} \Sigma_{g_{\mid J}}} .
\end{aligned}
$$

The last expression is purely Gaussian, and under the assumed conditions of nondegeneracy, also clearly finite, so we are done.

Before turning to concatenated-harmonisable processes we require a technical lemma.

Lemma 4. Let $A$ and $B$ be two $n \times n$ matrices, and suppose that $A$ is of rank $m \leq n$. Then each term in the standard Laplace expansion of $\operatorname{det}(A+B)$ involves at most $m$ elements of $A$.

Proof. We shall prove the result for $m=1$. The result for general $m$ then follows by writing a matrix of rank $m$ as the sum of $m$ matrices of rank 1 .

Since $A$ has rank 1 , there is a vector $a$ and numbers $\theta_{1}, \ldots, \theta_{n}$ such that the $j$ th row of $A$ is $\theta_{j} a, j=1, \ldots, n$. Let $b_{j}$ be the $j$ th row of $B$. Writing the determinant of $A+B$ as a function of its rows we have

$$
\begin{aligned}
\operatorname{det}(A+B) & =\operatorname{det}\left(\theta_{1} a+b_{1}, \theta_{2} a+b_{2}, \ldots, \theta_{n} a+b_{n}\right) \\
& =\theta_{1} \operatorname{det}\left(a, \theta_{2} a+b_{2}, \ldots, \theta_{n} a+b_{n}\right)+\operatorname{det}\left(b_{1}, \theta_{2} a+b_{2}, \ldots, \theta_{n} a+b_{n}\right) .
\end{aligned}
$$

Note that

$$
\begin{aligned}
\theta_{1} \operatorname{det} & \left(a, \theta_{2} a+b_{2}, \ldots, \theta_{n} a+b_{n}\right) \\
& =\theta_{1} \theta_{2} \operatorname{det}\left(a, a, \ldots, \theta_{n} a+b_{n}\right)+\theta_{1} \operatorname{det}\left(a, b_{2}, \ldots, \theta_{n} a+b_{n}\right) \\
& =\theta_{1} \operatorname{det}\left(a, b_{2}, \ldots, \theta_{n} a+b_{n}\right) \\
& =\cdots \\
& =\theta_{1} \operatorname{det}\left(a, b_{2}, b_{3}, \ldots, b_{n}\right) .
\end{aligned}
$$

Similarly,

$$
\operatorname{det}\left(b_{1}, \theta_{2} a+b_{2}, \ldots, \theta_{n} a+b_{n}\right)=\sum_{k=2}^{n} \theta_{k} \operatorname{det}\left(b_{1}, \ldots, b_{k-1}, a, b_{k+1}, \ldots, b_{n}\right),
$$

and continuing this process leads to

$$
\operatorname{det}(A+B)=\operatorname{det} B+\sum_{k=1}^{n} \theta_{k} \operatorname{det}\left(b_{1}, \ldots, b_{k-1}, a, b_{k+1}, \ldots, b_{n}\right),
$$

from which the result follows. 
We now have what we need to prove our last lemma.

Lemma 5. Let $f$ be a harmonisable, or concatenated-harmonisable, S $\alpha$ S random field satisfying the conditions of Theorem 3, or Theorem 4, respectively. Then $\mathrm{E}\left\{\left|\varphi\left(A_{u}(f, T)\right)\right|\right\}<\infty$ for every $u \in \mathbb{R}$.

Proof. We shall prove the result in the notation of the concatenated-harmonisable case, since taking $N^{\prime}=N$ gives the harmonisable case. In view of the argument leading to (51), and the structure of the conditional process $\hat{f}$ in this case (cf. the proof of Theorem 3 for this and the following notation) we need to show that

$$
\mathrm{E}\left\{\frac{\mathrm{E}\left\{\left|\operatorname{det} \sum_{k=1}^{\infty} \Gamma_{k}^{-1 / \alpha} G_{k} W_{k}\right|\right\}}{\left(\operatorname{det} \sum_{k=1}^{\infty} \Gamma_{k}^{-2 / \alpha} W_{k}\right)^{1 / 2}}\right\}<\infty,
$$

where the inner expectation is taken only with respect to the Gaussian random variables. Recall also that each $W_{k}$, given by (39), can be written in the form

$$
W_{k}=\sum_{l=1}^{N^{\prime}} W_{k, l}
$$

where $\left(W_{k, l}, k \geq 1, l=1, \ldots, N^{\prime}\right)$ are i.i.d., rank $1, n \times n$, random matrices of the form (32).

We concentrate on the denominator in (52) first. Set

$$
d=\left\lfloor\frac{n}{N^{\prime}}\right\rfloor,
$$

and recall that the determinant of the sum of nonnegative definite matrices is at least as large as the determinant of each of the terms, so that

$$
\begin{aligned}
& \operatorname{det}\left(\sum_{k=1}^{\infty} \Gamma_{k}^{-2 / \alpha} W_{k}\right) \\
& \quad \geq \operatorname{det}\left(\sum_{k=1}^{d} \Gamma_{k}^{-2 / \alpha} W_{k}+\Gamma_{d+1}^{-2 / \alpha}\left(W_{d+1,1}+\cdots+W_{d+1, n-d N^{\prime}}\right)\right) \\
& \quad=\Gamma_{d+1}^{-2\left(n-d N^{\prime}\right) / \alpha} \prod_{k=1}^{d} \Gamma_{k}^{-2 N^{\prime} / \alpha} \operatorname{det}\left(\sum_{k=1}^{d} W_{k}+\left(W_{d+1,1}+\cdots+W_{d+1, n-d N^{\prime}}\right)\right)
\end{aligned}
$$

the last line following from Lemma 4. Set

$$
D=\operatorname{det}\left(\sum_{k=1}^{d} W_{k}+\left(W_{d+1,1}+\cdots+W_{d+1, n-d N^{\prime}}\right),\right.
$$

so that, to prove (52), we need check only that

$$
\mathrm{E}\left\{\frac{\mathrm{E}\left\{\left|\operatorname{det} \sum_{k=1}^{\infty} \Gamma_{k}^{-1 / \alpha} G_{k} W_{k}\right|\right\}}{D \Gamma_{d+1}^{-2\left(n-d N^{\prime}\right) / \alpha} \prod_{k=1}^{d} \Gamma_{k}^{-2 N^{\prime} / \alpha}}\right\}<\infty .
$$


For later reference, note that $D$ is a polynomial function of $n$ i.i.d. random vectors, each of length $n$. Under the conditions of the theorem, the $n^{2}$ random variables defining $D$ have a bounded joint density with compact support in $\mathbb{R}^{n^{2}}$. We claim that it follows from this that there exists a finite $C$ such that, for small enough $\varepsilon$,

$$
\mathrm{P}\{D \leq \varepsilon\} \leq C \varepsilon
$$

In turn, from this, it trivially follows that

$$
\mathrm{E}\left\{D^{-1 / 2}\right\}<\infty
$$

To prove (54), first write $X_{1}, \ldots, X_{n}$ for the $n$ random vectors defining the determinant $D$, and let $A \subset \mathbb{R}^{n}$ denote the support of each one. Fix $\varepsilon>0$, and define

$$
\begin{aligned}
& A_{\varepsilon}=\left\{x=\left(x_{1}, \ldots, x_{n}\right) \in A^{n}: \min _{1 \leq i, j \leq n}\left|x_{i}(j)\right| \leq \varepsilon\right\}, \\
& B_{\varepsilon}=\left\{x=\left(x_{1}, \ldots, x_{n}\right) \in A^{n}: D\left(x_{1}, \ldots, x_{n}\right) \leq \varepsilon\right\} .
\end{aligned}
$$

Then

$$
\mathrm{P}\{D \leq \varepsilon\} \leq v\left(A_{\varepsilon}\right)+v\left(B_{\varepsilon} \cap\left(A^{n} \backslash A_{\varepsilon}\right)\right),
$$

where $v$ is the measure on $A^{n}$ generated by the random variables $X_{1}, \ldots, X_{n}$. Both of these terms are easily seen to be bounded by a constant multiple of $\varepsilon$, if $\varepsilon$ is small enough. The first is small, since $A_{\varepsilon}$ can be covered by a finite number of $n$-dimensional rectangles, one of whose sides has length no more than $2 \varepsilon$ and the remaining sides having length no more than the diameter of $A$. Since $v$ has bounded density, this gives that $\mu\left(A_{\varepsilon}\right) \leq C \varepsilon$ for some finite $C$.

As far as the second term in (56) is concerned, we note that, because of the smoothness of the mapping $D: \mathbb{R}^{n^{2}} \rightarrow \mathbb{R}$ in the region $A^{n} \backslash A_{\varepsilon}$, the set $B_{\varepsilon} \cap\left(A^{n} \backslash A_{\varepsilon}\right)$ is a $C^{\infty}$, locally convex, stratified manifold, and so it follows from the generalized tube formula of [2, Theorem 10.9.5] that this set also has $\nu$-measure bounded by $C \varepsilon$.

Returning now to (53), note that, by expanding the determinant and applying Lemma 4, we find that (53) is equivalent to

$$
\mathrm{E}\left\{\frac{\mathrm{E}\left\{\left|\sum_{k_{1}=1}^{\infty} \cdots \sum_{k_{n}=1}^{\infty} \Gamma_{k_{1}}^{-1 / \alpha} \cdots \Gamma_{k_{n}}^{-1 / \alpha} G_{k_{1}} \cdots G_{k_{n}} B_{k_{1} \cdots k_{n}} \mathbf{1}_{F}\right|\right\}}{D \Gamma_{d+1}^{-2\left(n-d N^{\prime}\right) / \alpha} \prod_{k=1}^{d} \Gamma_{k}^{-2 N^{\prime} / \alpha}}\right\},
$$

where the $B_{k_{1} \cdots k_{n}}$ are uniformly bounded random variables, independent of $\Gamma_{j}$ and the Gaussian $G_{j}$, and $F$ is the event that at most $N^{\prime}$ of $k_{1}, \ldots, k_{n}$ are equal.

Fixing the $\Gamma_{j}$, applying the boundedness of the $B_{k_{1} \cdots k_{n}}$, taking an expectation over the Gaussian $G_{j}$, and using their symmetry, bounds the above by

$$
C \mathrm{E}\left\{\left(\frac{1}{D} \sum_{k_{1}=1}^{\infty} \cdots \sum_{k_{n}=1}^{\infty} \frac{\Gamma_{k_{1}}^{-2 / \alpha} \cdots \Gamma_{k_{n}}^{-2 / \alpha}}{\Gamma_{d+1}^{-2\left(n-d N^{\prime}\right) / \alpha} \prod_{k=1}^{d} \Gamma_{k}^{-2 N^{\prime} / \alpha}} \mathbf{1}_{F}\right)^{1 / 2}\right\},
$$

where $C$ is a finite constant which may change from line to line. Now use the fact that $D$ is independent of all the other random variables in the above expectation, along with (55), to remove the factor of $D^{-1}$ from the expectation, with an appropriate change of the constant $C$. 
We may, and shall, assume that, in the $k$-fold sum in (57), $k_{1} \leq k_{2} \leq \cdots \leq k_{n}$. Thus, what remains to show is that the remaining expectation, which we rewrite as

$$
\mathrm{E}\left\{\left(\sum_{k_{1}=1}^{\infty} \cdots \sum_{k_{n-1}=k_{n-2}+1}^{\infty} \frac{\Gamma_{k_{1}}^{-2 / \alpha} \cdots \Gamma_{k_{n-1}}^{-2 / \alpha}}{\Gamma_{d+1}^{-2\left(n-d N^{\prime}-1\right) / \alpha} \prod_{k=1}^{d} \Gamma_{k}^{-2 N^{\prime} / \alpha}} \sum_{k_{n}=k_{n-1}+1}^{\infty} \Gamma_{d+1}^{2 / \alpha} \Gamma_{k_{n}}^{-2 / \alpha} \mathbf{1}_{F}\right)^{1 / 2}\right\},
$$

is finite. Consider the summation over $k_{n}$, keeping $k_{1}, \ldots, k_{n-1}$ fixed. Since each term in the ( $n$-fold) sum is bounded from above by 1 , we can begin the summation over $k_{n}$ at an arbitrary large value $K$. Then, the expectation in the last sum is bounded by

$$
\mathrm{E}\left\{\left(\Gamma_{d+1}^{2 / \alpha} \sum_{k_{n}=K}^{\infty} \Gamma_{k_{n}}^{-2 / \alpha}\right)^{1 / 2}\right\}
$$

Since gamma random variables have all moments finite, by Hölder's inequality, it is enough to prove that, for $\varepsilon \leq 1$,

$$
\mathrm{E}\left\{\left(\sum_{k_{n}=K}^{\infty} \Gamma_{k_{n}}^{-2 / \alpha}\right)^{(1+\varepsilon) / 2}\right\}<\infty
$$

Furthermore, since it is easy to check that

$$
\mathrm{E}\left\{\Gamma_{k}^{-2 / \alpha}\right\} \leq C k^{-2 / \alpha}
$$

for large enough $k$, the finiteness of (58) now follows from the fact that the expectation in (59) is bounded by

$$
\left(\sum_{k_{n}=K}^{\infty} \mathrm{E}\left\{\Gamma_{k_{n}}^{-2 / \alpha}\right\}\right)^{(1+\varepsilon) / 2}
$$

and that $\alpha<2$.

Next, we consider the double sums obtained by fixing $k_{1}, \ldots, k_{n-2}$ and taking each sum only over $k_{n-1}$ and $k_{n}$. As in the case we have just considered, we can start the sum over $k_{n-1}$ at a (large) $K$ of our choice, and then the expectation involving the double summation, with an additional factor of $\Gamma_{d+1}^{2 / \alpha}$ brought into play, is bounded above by

$$
\mathrm{E}\left\{\left(\Gamma_{d+1}^{4 / \alpha} \sum_{k_{n-1}=K}^{\infty} \sum_{k_{n}=K}^{\infty} \Gamma_{k_{n-1}}^{-2 / \alpha} \Gamma_{k_{n}}^{-2 / \alpha}\right)^{1 / 2}\right\} .
$$

The finiteness of this expression follows as above, from the easily checkable fact that

$$
\mathrm{E}\left\{\Gamma_{k_{1}}^{-2 / \alpha} \Gamma_{k_{2}}^{-2 / \alpha}\right\} \leq C k_{1}^{-2 / \alpha} k_{2}^{-2 / \alpha}
$$

for all large enough $k_{1} \leq k_{2}$. Iterating this argument, we arrive at the finiteness of (57), once we prove that, for all large enough $k_{1} \leq k_{2} \leq \cdots \leq k_{n}$,

$$
\mathrm{E}\left\{\prod_{j=1}^{n} \Gamma_{k_{j}}^{-2 / \alpha}\right\} \leq C \prod_{j=1}^{n} j^{-2 / \alpha} .
$$


This, however, follows from Hölder's inequality and the fact that, for all large enough $k$,

$$
\mathrm{E}\left\{\Gamma_{k}^{-2 n / \alpha}\right\} \leq C k^{-2 n / \alpha} .
$$

This completes the argument.

Lemma 6. The exchange of expectation and integration in (48) is justified.

Proof. In the notation of (48), it will suffice to show that

$$
\int_{\operatorname{Graff}(N, N-j)} \mathrm{E}\left\{\left|\mathcal{L}_{0}\left(A_{u}(f, M) \cap V\right)\right|\right\} \mathrm{d} \lambda_{N-j}^{N}(V)<\infty .
$$

Note first that (48) was only being applied under the conditions of one of the main theorems of the paper, so we know that the inner expectation is always finite. We also know, from the discussions in this appendix that, for any convex $A$,

$$
\left|\mathcal{L}_{0}\left(A_{u}(f, A)\right)\right| \leq \psi(A),
$$

where we define $\psi(A)$ to be the number of critical points of $f$ in $A$ and on its various boundaries. All of these also have finite expectations. The functional $\mathrm{E}\{\psi\}$ is clearly additive (since $\psi$ itself is) in the sense of (5), as well as being invariant under rigid motions (by isotropy) and continuous in the Hausdorff metric. Thus, by Hadwiger's theorem (cf. (6)), there exist constants $c_{j}$, dependent of $u$ and the structure of $f$, such that

$$
\mathrm{E}\{\psi\}=\sum_{k=0}^{N} c_{k} \mathcal{L}_{k}(A) .
$$

Substituting into (60) we therefore have

$$
\begin{aligned}
& \int_{\operatorname{Graff}(N, N-j)} \mathrm{E}\left\{\left|\mathcal{L}_{0}\left(A_{u}(f, M) \cap V\right)\right|\right\} \mathrm{d} \lambda_{N-j}^{N}(V) \\
& \leq \int_{\operatorname{Graff}(N, N-j)} \mathrm{E}\{\psi(M \cap V)\} \mathrm{d} \lambda_{N-j}^{N}(V) \\
& =\int_{\operatorname{Graff}(N, N-j)} \sum_{k=0}^{N} c_{k} \mathcal{L}_{k}(M \cap V) \mathrm{d} \lambda_{N-j}^{N}(V) \\
& =\sum_{k=0}^{N} c_{k} \int_{\operatorname{Graff}(N, N-j)} \mathcal{L}_{k}(M \cap V) \mathrm{d} \lambda_{N-j}^{N}(V) \\
& =\sum_{j=0}^{N} c_{j}\left[\begin{array}{c}
k+j \\
k
\end{array}\right] \mathcal{L}_{k+j}(M),
\end{aligned}
$$

the last line following from Crofton's formula (46). Since $M$ is compact and convex, the $\mathcal{L}_{j}(M)$ are all finite, and so we are done.

\section{References}

[1] AdLer, R. ANd SAmorodnitsky, G. (1997). Level crossings of absolutely continuous stationary symmetric $\alpha$-stable processes. Ann. Appl. Prob. 7, 460-493.

[2] Adler, R. J. And Taylor, J. E. (2007). Random Fields and Geometry. Springer, New York. 
[3] Adler, R. J., Samorodnitsky, G. and Gadrich, T. (1993). The expected number of level crossings for stationary, harmonisable, symmetric, stable processes. Ann. Appl. Prob. 3, 553-575.

[4] Adler, R. J., TAYlor, J. AND Worsley, K. (2010). Random Fields and Geometry: Applications. In preparation. Early chapters available at http://webee.technion.ac.il/people/adler/publications.html.

[5] Breiman, L. (1965). On some limit theorems similar to the arc-sine law. Theory Prob. Appl. 10, 323-331.

[6] Hadwiger, H. (1957). Vorlesüngen Über Inhalt, Oberfläche und Isoperimetrie. Springer, Berlin.

[7] Klain, D. A. And Rota, G.-C. (1997). Introduction to Geometric Probability. Cambridge University Press.

[8] Samorodnitsky, G. And Taqqu, M. S. (1994). Stable Non-Gaussian Random Processes. Chapman and Hall, New York.

[9] Worsley, K. (1997). The geometry of random images. Chance 9, 27-40. 